\title{
Progression of renal fibrosis: the underestimated role of endothelial alterations
}

\author{
Dominique Guerrot ${ }^{1,2,3}$, Jean-Claude Dussaule ${ }^{1,2}$, Panagiotis Kavvadas ${ }^{1}$, Jean-Jacques Boffa ${ }^{1,2}$, \\ Christos E Chadjichristos ${ }^{1,2}$, Christos Chatziantoniou ${ }^{1,2^{*}}$ \\ From Fibroproliferative disorders: from biochemical analysis to targeted therapies \\ Frauenchiemsee, Germany. 25-30 September 2010
}

\begin{abstract}
The vasculature of the kidney is a heterogeneous structure, whose functional integrity is essential for the regulation of renal function. Owing to the importance of the endothelium in vascular biology, chronic endothelial alterations are therefore susceptible to impair multiple aspects of renal physiology and, in turn, to contribute to renal fibrosis. Although systemic endothelial dysfunction is undoubtedly associated with chronic kidney disease, the role of the renal endothelium in the initiation and the progression of renal fibrosis remains largely elusive. In this article, we critically review recent evidence supporting direct and indirect contributions of renal endothelial alterations to fibrosis in the kidney. Specifically, the potential implications of renal endothelial dysfunction and endothelial paucity in parenchymal hypoxia, in the regulation of local inflammation, and in the generation of renal mesenchymal cells are reviewed. We thereafter discuss therapeutic perspectives targeting renal endothelial alterations during the initiation and the progression of renal fibrogenesis.
\end{abstract}

\section{Introduction}

The kidney receives approximately $20 \%$ of the cardiac output, and many essential functions of the organ are supported by the complex organization of renal microvasculature. Therefore primitive or secondary pathological changes in arterioles, glomerular capillaries, vasa rectae and/or peritubular capillaries are susceptible to impair different aspects of renal physiology and, in turn, to contribute to the progression of chronic kidney disease (CKD). Endothelial cells constitute the inner lining of the vessels and are a cornerstone of vascular homeostasis. Besides its classical barrier function, the endothelium is a key player in physiological processes such as the regulation of vasomotor tone, the control of tissue inflammation and of thrombosis $[1,2]$. Within the renal microvasculature, the endothelium is characterized by a remarkable structural heterogeneity, related to the different and highly specialized functions of endothelial cells, from the preglomerular arterioles to the peritubular capillary bed.

\footnotetext{
* Correspondence: christos.chatziantoniou@upmc.fr

'INSERM U702, Tenon Hospital, Paris, France

Full list of author information is available at the end of the article
}

The term "endothelial dysfunction" has been used to define diverse syndromes characterized by changes in distinct endothelial functions, related to a cellular phenotypic switch from a quiescent to an activated state. No clear definition of endothelial dysfunction has been established so far, and this multifaceted disorder actually encompasses a spectrum of disturbances in vasomotor responses, antithrombogenic properties, vascular permeability, leukocyte recruitment and endothelial cell proliferation. In the clinical setting endothelial dysfunction may be detected non-invasively by functional tests evaluating the vasomotor effects of pharmacological substances such as acetylcholine, or of flow-mediated vasodilation after transient ischemia on distal conduit arteries [3,4]. Considerable interest has also been focused on the identification of circulating markers associated with endothelial dysfunction. These include endothelin 1 (ET-1), metabolites of NO (nitrites, nitrates), markers of fibrinolysis and anticoagulant activity (plasminogen activator inhibitor 1, soluble thrombomodulin), and soluble endothelial adhesion molecules (s-E-selectin, s-ICAM, s-VCAM) [5]. More recently circulating endothelial cells, endothelial microparticles and 
endothelial progenitor cells have been proposed as alternative markers of endothelial cell dysfunction [6].

Cardiovascular outcomes are the major cause of death in end-stage renal disease patients [7]. During the past decade endothelial dysfunction has emerged as an important intermediate factor in CKD. Indeed, with the decreasing glomerular filtration rate, the vasculature is progressively exposed to a burden of pathogenetic conditions responsible for severe functional changes in the endothelium, such as reactive oxygen species (ROS), assymetrical dymethylarginine (ADMA), homocysteine or glycosylated end products [8-11]. We and others have identified ADMA, an endogenous inhibitor of NO synthase (NOS) elevated in CKD patients, as a mediator of endothelial dysfunction, oxidative stress and fibrogenesis [12,13]. Oxidative stress plays an important role in cellular responses to injury, and is a central process in the pathophysiology of endothelial dysfunction. In endothelial cells, ROS can be generated by uncoupled eNOS, which normally produces NO, and lead to the production of oxygen peroxide and subsequent modifications of the cellular phenotype [2,14].

Although the recognition of a systemic endothelial disease related to CKD has led to significant research interest, fewer studies have specifically focused on endothelial alterations within the diseased kidney. We have shown that pharmacological NO deficiency led to ET-1 production in the injured renal endothelial cells with direct profibrotic consequences in the kidney [15]. Recent evidence provides novel insights on the pathophysiological role of intrarenal endothelium in the progression of CKD (Figure 1). In this review we analyze direct and indirect consequences of endothelial alterations on hemodynamics, inflammation and fibrogenesis in the kidney, and discuss therapeutic issues targeting this underestimated culprit in renal fibrosis.

\section{Review}

\section{Renal endothelial injury contributes to parenchymal hypoxia}

Chronic hypoxia mediates the progression of renal fibrosis, even from the early stages of CKD [16]. Interstitial fibroblasts, epithelial cells and endothelial cells develop different responses to hypoxia, which may directly or indirectly contribute to profibrotic mechanisms. Human renal fibroblasts exposed to experimental hypoxic conditions increase collagen production and decrease the expression of extracellular matrix remodeling enzymes [17]. Aerobic oxidative metabolism-dependent epithelial cells physiologically adapt to a reduction in oxygen tension by increasing HIF (hypoxia inducible factor)-dependent signaling, which in turn promotes cell survival [18]. In chronic hypoxic conditions these tubular adaptive mechanisms may be overrun and cell function and viability compromised.
Although the endothelium receives direct oxygen supply from red blood cells, global or regional hemodynamic disturbances may also be responsible for endothelial hypoxia and activate the endothelial cells. In a rat model of renal artery clamping with rescue by non-injured endothelial cells, Brodsky and colleagues identified acute endothelial dysfunction as a contributor to the hemodynamic "no-reflow" phenomenon in post-ischemic kidneys [19]. In this setting, endothelial activation and subsequent adhesion of leukocytes to the endothelium was believed to create hemodynamic resistances which reduce regional blood flow.

Peritubular capillaries constitute the major network supplying oxygen to the nephrons. Therefore capillary functional alterations, by reducing blood flow or leading to subsequent reduction of vascular density, are susceptible to be a major determinant of kidney disease progression. Consistently, studies on human biopsies have associated microvascular rarefaction with the progression of renal failure, while evidence for a pathogenetic role of capillary endothelial alterations in CKD progression via chronic tubulointerstitial hypoxia stems from experimental models. In renal biopsies of CKD patients, Choi and colleagues have demonstrated that morphological alterations of peritubular capillaries were strongly associated with parameters of tubulointerstitial injury [20]. Similarly a histomorphological evaluation of cadaveric kidney allografts performed at the time of transplantation showed that sustained kidney injury is associated with a reduction in Von Willebrand Factor staining on peritubular capillaries (but not on renal arteries or arterioles) [21]. In experimental renovascular disease therapeutic prevention of vascular rarefaction by intrarenal administration of VEGF leads to the preservation of renal blood flow and a reduction in subsequent fibrosis [22]. Together with several other studies, these results suggest straightforward pathophysiological consequences of peritubular endothelial alterations on both short- and long-term aspects of renal function.

Interlobular arteries and afferent arterioles are the major site for renal blood flow autoregulation. Since endothelial cells control vasomotor tone in these resistance vessels, in particular via $\mathrm{NO}$ and endothelium-derived hyperpolarizing factors, alterations of the endothelium may also contribute to glomerular hemodynamic disturbances and to downstream hypoxia in the kidney. Fawn-Hooded hypertensive (FHH) rats present defective renal blood flow autoregulation and develop progressive kidney failure with age. Ochodnicky and colleagues have studied endotheliumdependent vasodilation in this inbred model of spontaneous renal disease and identified that endothelial dysfunction in the renal artery precedes the development of kidney damage [23]. Although the mechanistic explanations for these results presumably differ from those related 


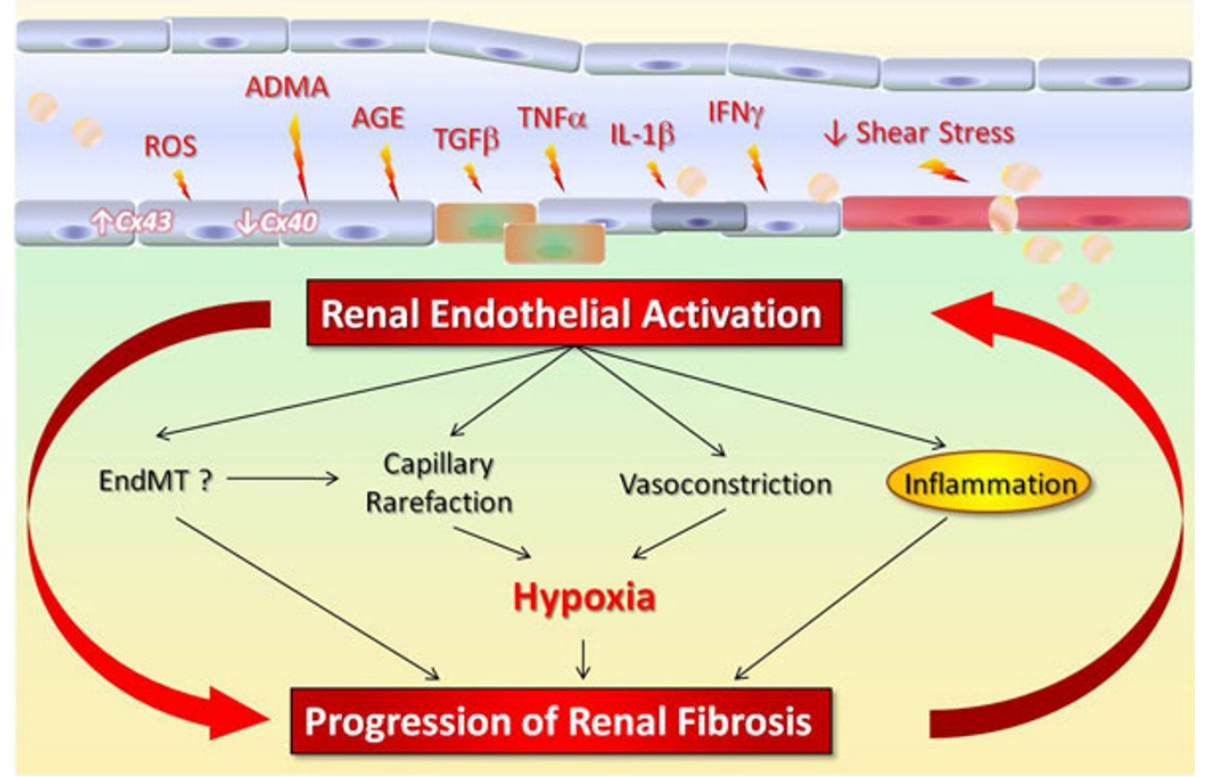

Figure 1 Schematic view of the pathophysiological role of endothelial activation in chronic kidney disease progression. (ADMA assymetrical dymethylarginine; ROS reactive oxygen species; AGE advanced glycation end products; TGF transforming growth factor; TNF tumor necrosis factor; IL interleukin; IFN interferon; EndMT endothelial-mesenchymal transition; Cx40 Connexin 40: Cx43 Connexin 43.)

to chronic peritubular endothelium injury, this study establishes that the integrity of preglomerular endothelium is required for renal vascular homeostasis.

Overall, current evidence underlines renal endothelial hypoxia as the actor of an important profibrotic vicious circle in CKD progression (Figure 1).

\section{Endothelial functional alterations promote tissue inflammation}

Exaggerated inflammation has long been identified as a major player in acute and chronic renal diseases, including allograft rejection, glomerulonephritis, ischemia-reperfusion or cisplatin-induced nephropathy. The endothelium is at the interface between blood and circulating cells on the one hand, and tissue on the other hand. In physiological and pathological conditions, endothelial cells integrate mechanical stimuli related to local hemodynamics and cytokinic stimuli such as IFN-gamma, IL-1beta or TNFalpha. The latter cytokines are able to induce the expression of distinct patterns of adhesion molecules on the luminal surface, thereby promoting a site- and cell-specific recruitment of circulating leukocytes. Schematically leukocyte recruitment is a three-step process. Leukocytes first roll on the activated endothelium, via the interaction between selectins (E-selectin, P-selectin) and leukocyte surface antigens. Firm adhesion is then facilitated by the endothelial expression of ICAM-1 and VCAM-1, which also promote subsequent transmigration of the leukocytes into the tissue, together with PECAM/CD31.
In the kidney pathophysiological implications of endothelial selectins and adhesion molecules have been demonstrated in a large variety of clinical settings and experimental models. In an ischemic model of renal failure, P-selectin blockade with monoclonal antibodies improved renal function and the administration of fucoidan, an oligosaccharide which non-specifically inhibits P-selectin, increased renal blood flow [24]. Similarly, transgenic mice lacking basigin/CD147, a ligand for E-selectin, exhibited reduced kidney damage after ischemia-reperfusion as compared to control mice, indicating a critical role for E-selectin in neutrophil recruitment in this setting [25]. In rodents, the injection of allo-immune nephrotoxic serum induced the expression of endothelial selectins and leukocyte rolling in glomeruli and capillaries, an event that was significantly blunted by fucoidan in postcapillary venules $[26,27]$.

By using anti-ICAM-1 monoclonal antibodies in rats and ICAM-1 transgenic mice, Kelly and colleagues have shown that this adhesion molecule plays an early and important pathogenetic role in renal ischemia-reperfusion, via neutrophil infiltration, with both functional and structural consequences on the kidney $[28,29]$. Adhesion of circulating cells to the endothelium of renal vessels may also contribute to potentially severe hemodynamic alterations, which can further aggravate tissue injury. Overall these studies underline the importance of endothelial activation in the genesis of local inflammation at early stages of inflammatory kidney diseases. 
Gap-junctional intercellular communication is essential in the coordination and integration of microvascular function by the endothelial cells in a very complex manner. Gap junctions are composed of intercellular channels formed by connexins $(\mathrm{Cx})$, which allow the direct exchange of ions, small metabolites and other second messenger molecules between adjacent cells [30]. Each type of $\mathrm{Cx}$-made channel has a unique inherent gating property, or permeability to various molecules. Therefore, Cx composition of gap junction channels appears to determine selectivity among second messengers [31].

Alterations of the expression of endothelial $\mathrm{Cx}$ have been associated to the development of chronic and acute vascular inflammatory diseases [32]. For example, a decrease in the expression of $\mathrm{Cx} 40$ has been reported in the endothelial layer covering atherosclerotic lesions in the aorta of hypercholesterolemic mice. Mice in which Cx40 was specifically deleted from the endothelium showed enhanced monocyte infiltration at the very early stages of the disease [33]. In contrast to $\mathrm{Cx} 40, \mathrm{Cx} 43$ expression seems to be upregulated in the dysfunctional endothelium and endothelium-specific deletion of $\mathrm{Cx} 43$ in mice greatly reduced the TNF- $\alpha$-induced leukocyte adhesion and transmigration [34]. It has also been recently suggested that this $\mathrm{Cx}$ may serve as a conducting pathway by amplifying $\mathrm{Ca}^{2+}$-signalling between endothelial cells to spread inflammatory signals within the lung capillary network [35]. In addition, $\mathrm{Cx} 43^{+/-}$mice were protected against chronic or acute inflammatory disease, as they displayed reduced inflammatory cell recruitment at the injured site [32]. Interestingly, in accordance with the above mentioned studies, we recently observed a marked increase of the expression of $\mathrm{Cx} 43$ in the endothelium of peritubular and glomerular capillaries at the early stages of hypertension-induced renal disease in mice. The Cx43 expression pattern was paralleled closely by that of adhesion markers such as VCAM-1 and ICAM-1, known to play a major role in the recruitment of inflammatory cells [36]. Although further work is required to clarify the implication of $\mathrm{Cx}$ in renal inflammation, the $\mathrm{Cx}$ hypothesis may be of interest in the recruitment of inflammatory cells in the kidney, as several studies showed an important role of these proteins in the inflammatory response.

\section{Endothelial-mesenchymal transition: a new player in renal fibrosis?}

The origin of renal fibroblasts is an evolving and somewhat controversial issue [37-39]. Acquisition of a mesenchymal-like phenotype by tubular epithelial cells, named epithelial-mesenchymal transition (EMT), has been largely studied in renal fibrosis over the past decade. More recently several research teams have suggested that endothelial cells may also acquire functional and structural characteristics of mesenchymal cells after tissue injury. This so-called endothelial-mesenchymal transition (EndMT) had previously been recognized in normal conditions, particularly during embryonic development of the heart. Increasing evidence now argues that EndMT may play an additional role in a variety of diseases, including in kidney injury [40].

In vitro, endothelial cells of different origins can acquire a mesenchymal phenotype, spontaneously or after stimulation by TGF beta. In a model of cardiac overload in Tie2Cre;ROSA-STOP-lacZ transgenic mice, the presence of markers usually expressed by myofibroblasts (FSP-1, alpha SMA) was partially co-localized with the endothelial fate tracer lacZ, suggesting the endothelial origin of heart myofibroblasts [41]. Interestingly mice treated with human recombinant BMP-7 appeared to be protected against both EndMT and the development of cardiac fibrosis.

During the past 4 years, an EndMT-like process has also been evidenced in the kidney in several independent experimental models (unilateral ureteral obstruction, pharmacological inhibition of eNOS, streptozotocin-induced nephropathy, Col4a3-deficient mice) [42-44]. We have also evaluated the modifications of endothelial-cell phenotype during the progression of angiotensin II-induced nephropathy in Sprague Dawley rats, and observed a limited and focal co-expression of FSP1 with the endothelial marker RECA1, which suggests the presence of EndMT in peritubular renal capillaries in hypertensive nephropathy. Interestingly, features consistent with EndMT were observed at early stages of the renal disease, before the onset of significant proteinuria or fibrosis (Guerrot et al., unpublished data). Recently, Basile and collegues have analyzed the phenotypic alterations of endothelial cells after ischemia-reperfusion in Tie2-Cre;YFP transgenic mice [45]. The results of this study suggested that the reduced density of endothelial cells following renal ischemia may be due, in part, to EndMT.

Together, these data identify EndMT as a novel aspect of endothelial dysfunction and show that EndMT may be associated with kidney disease. Furthermore, they suggest that endothelial cells which acquire mesenchymal characteristics may directly contribute to early pathogenetic mechanisms of fibrogenesis. However, owing to the heterogeneity of renal endothelial cells, further studies would be of major interest to better understand the relative importance, the molecular mechanisms and local consequences of EndMT, especially with respect to the different compartments of renal microvasculature.

\section{Targeting renal endothelial dysfunction as an innovative therapeutical approach in renal fibrosis}

Since current evidence underlines the importance of endothelial alterations in CKD progression, the prevention of renal endothelial injury emerges as a promising treatment strategy in kidney diseases. Depending on the clinical 
setting a reduction of endothelial dysfunction is susceptible to alleviate inflammation, hemodynamic disturbances, hypoxia and extracellular matrix synthesis. In renal experimental models, interesting studies have suggested beneficial effects of therapies based on selectin inhibitors, ET-1 antagonists or treatments increasing $\mathrm{NO}$ bioavailability [15,46-48]. These strategies all target distinct aspects of endothelial pathophysiology, thereby reducing specific consequences of renal endothelial functional alterations. In a different approach several teams have also shown promising results when promoting replacement of the injured endothelium, either by increasing resident endothelial cell proliferation, stimulating endogenous progenitor mobilization or directly injecting autologous endothelial progenitor cells into the kidney [49-51].

An important characteristic of endothelial activation in kidney diseases is de novo expression of surface antigens. Importantly, critical modifications of the endothelial phenotype predominantly occur in the injured region. Therefore, specifically targeting the activated endothelial cells may allow selective delivering of drugs to the diseased vascular bed. This strategy has proven to be efficient in preliminary studies using liposomes conjugated to anti-Eselectin antibodies, to address dexamethasone in a model of allo-immune nephrotoxic serum-induced renal disease $[27,48]$.

\section{Conclusion}

Endothelial dysfunction is a multifaceted disorder that plays a central role in complications related to kidney diseases. Beside the well established systemic endothelial dysfunction associated with CKD, recent evidence highlights direct implications of renal endothelial activation in fibrogenesis. Together these data suggest that the altered renal endothelium may be a promising therapeutic target and prompt further studies to evaluate novel specific strategies for the treatment of CKD progression. Specifically, the possibility of targeted delivery based on endothelial alterations opens novel therapeutic avenues for the treatment of renal diseases. Future research directions may also include inhibition of EndMT, preservation of endothelium-derived hyperpolarizing factors, pharmacological maintenance of eNOS activation in altered shear stress conditions, and silencing of endothelial proinflammatory genes.

\footnotetext{
Acknowledgements

This article has been published as part of Fibrogenesis \& Tissue Repair Volume 5 Supplement 1, 2012: Proceedings of Fibroproliferative disorders: from biochemical analysis to targeted therapies. The full contents of the supplement are available online at http://www.fibrogenesis.com/ supplements/5/S1.

\section{Author details}

${ }^{1}$ INSERM U702, Tenon Hospital, Paris, France. ${ }^{2}$ Université Pierre et Marie Curie, Paris, France. ${ }^{3}$ Service de Néphrologie, CHU Hôpitaux de Rouen, Rouen, France.
}

\section{Authors' contributions}

DG drafted the manuscript. JCD, JJB, CEC and CC helped to draft the manuscript. DG and CC revised the manuscript. All authors read and approved the final manuscript.

\section{Competing interests}

The authors declare no conflict of interest regarding the issues addressed in this review.

Published: 6 June 2012

\section{References}

1. Félétou M, Vanhoutte PM: Endothelial dysfunction: a multifaceted disorder (The Wiggers Award Lecture). Am J Physiol Heart Circ Physiol 2006, 291:H985-1002

2. Deanfield JE, Halcox JP, Rabelink TJ: Endothelial function and dysfunction: testing and clinical relevance. Circulation 2007, 115:1285-1295.

3. Joannides $R$, Haefeli $W E$, Linder $L$, et al: Nitric oxide is responsible for flow-dependent dilatation of human peripheral conduit arteries in vivo. Circulation 1995, 91:1314-1319.

4. Thuillez C, Richard V: Targeting endothelial dysfunction in hypertensive subjects. J Hum Hypertens 2005, 19 Suppl 1: S21-25.

5. Constans J, Conri C: Circulating markers of endothelial function in cardiovascular disease: Clin Chim Acta. Netherlands 2006, 33-47.

6. Rabelink TJ, de Boer HC, van Zonneveld AJ: Endothelial activation and circulating markers of endothelial activation in kidney disease. Nat Rev Nephrol 2010, 6:404-414.

7. Go AS, Chertow GM, Fan D, McCulloch CE, Hsu CY: Chronic kidney disease and the risks of death, cardiovascular events, and hospitalization. N Engl J Med 2004, 351:1296-1305.

8. Zoccali C: Endothelial dysfunction in CKD: a new player in town? Nephrol Dial Transplant 2008

9. Kaysen GA, Eiserich JP: The role of oxidative stress-altered lipoprotein structure and function and microinflammation on cardiovascular risk in patients with minor renal dysfunction. J Am Soc Nephrol 2004, 15:538-548,

10. Cooke JP: Asymmetrical dimethylarginine: the Uber marker? Circulation 2004, 109:1813-1818.

11. Stam F, van Guldener C, Schalkwijk CG, ter Wee PM, Donker AJ, Stehouwer CD: Impaired renal function is associated with markers of endothelial dysfunction and increased inflammatory activity. Nephrol Dial Transplant 2003, 18:892-898.

12. Mihout $F$, Shweke N, Bige N, et al: Asymmetric dimethylarginine (ADMA) induces chronic kidney disease through a mechanism involving collagen and TGF-beta1 synthesis. J Pathol 2011, 223:37-45.

13. Fliser D, Kronenberg F, Kielstein JT, et al: Asymmetric dimethylarginine and progression of chronic kidney disease: the mild to moderate kidney disease study. J Am Soc Nephrol 2005, 16:2456-2461.

14. Bedard K, Krause KH: The NOX family of ROS-generating NADPH oxidases: physiology and pathophysiology. Physiol Rev 2007, 87:245-313.

15. Tharaux PL, Chatziantoniou C, Casellas D, Fouassier L, Ardaillou R, Dussaule JC: Vascular endothelin-1 gene expression and synthesis and effect on renal type I collagen synthesis and nephroangiosclerosis during nitric oxide synthase inhibition in rats. Circulation 1999, 99:2185-2191.

16. Mimura I, Nangaku M: The suffocating kidney: tubulointerstitial hypoxia in end-stage renal disease. Nat Rev Nephrol 2010, 6:667-678.

17. Norman JT, Clark IM, Garcia PL: Hypoxia promotes fibrogenesis in human renal fibroblasts. Kidney Int 2000, 58:2351-2366.

18. Leonard MO, Cottell DC, Godson C, Brady HR, Taylor CT: The role of HIF-1 alpha in transcriptional regulation of the proximal tubular epithelial cell response to hypoxia. J Biol Chem 2003, 278:40296-40304.

19. Brodsky SV, Yamamoto T, Tada T, et al: Endothelial dysfunction in ischemic acute renal failure: rescue by transplanted endothelial cells. Am J Physiol Renal Physiol 2002, 282:F1140-1149.

20. Choi YJ, Chakraborty S, Nguyen V, et al: Peritubular capillary loss is associated with chronic tubulointerstitial injury in human kidney: altered expression of vascular endothelial growth factor. Hum Pathol 2000, 31:1491-1497.

21. Kwon O, Hong SM, Sutton TA, Temm CJ: Preservation of peritubular capillary endothelial integrity and increasing pericytes may be critical to 
recovery from postischemic acute kidney injury. Am J Physiol Renal Physiol 2008, 295:F351-359.

22. Hiescu R, Fernandez SR, Kelsen S, Maric C, Chade AR: Role of renal microcirculation in experimental renovascular disease. Nephrol Dial Transplant 2010, 25:1079-1087.

23. Ochodnický P, Henning RH, Buikema HJ, de Zeeuw D, Provoost AP, van Dokkum RP: Renal vascular dysfunction precedes the development of renal damage in the hypertensive Fawn-Hooded rat. Am J Physiol Renal Physiol 2010, 298:F625-633.

24. Bojakowski K, Abramczyk P, Bojakowska M, Zwolinska A, Przybylski J, Gaciong Z: Fucoidan improves the renal blood flow in the early stage of renal ischemia/reperfusion injury in the rat. J Physiol Pharmacol 2001, 52:137-143.

25. Kato N, Yuzawa Y, Kosugi T, et al: The E-selectin ligand basigin/CD147 is responsible for neutrophil recruitment in renal ischemia/reperfusion. $J$ Am Soc Nephrol 2009, 20:1565-1576.

26. De Vriese AS, Endlich $K$, Elger $M$, et al: The role of selectins in glomerular leukocyte recruitment in rat anti-glomerular basement membrane glomerulonephritis. J Am Soc Nephrol 1999, 10:2510-2517.

27. Asgeirsdóttir SA, Zwiers PJ, Morselt HW, et al: Inhibition of proinflammatory genes in anti-GBM glomerulonephritis by targeted dexamethasone-loaded AbEsel liposomes. Am J Physiol Renal Physiol 2008, 294:F554-561.

28. Kelly K, Williams WW Jr, Colvin RB, Bonventre JV: Antibody to intercellular adhesion molecule 1 protects the kidney against ischemic injury. Proc Natl Acad Sci USA 1994, 91:812-816.

29. Kelly KJ, Williams WW Jr, Colvin RB, et al: Intercellular adhesion molecule1-deficient mice are protected against ischemic renal injury. J Clin Invest 1996, 97:1056-1063.

30. Sohl G, Willecke K: Gap junctions and the connexin protein family. Cardiovasc Res 2004, 62:228-232.

31. Harris AL: Connexin channel permeability to cytoplasmic molecules. Prog Biophys Mol Biol 2007, 94:120-143.

32. Scheckenbach KE, Crespin S, Kwak BR, Chanson M: Connexin ChannelDependent Signaling Pathways in Inflammation. J Vasc Res Basel 2010, 91-103.

33. Chadjichristos $C E$, Scheckenbach $K E$, van Veen $T A$, et al: Endothelial-specific deletion of connexin 40 promotes atherosclerosis by increasing CD73dependent leukocyte adhesion. Circulation 2010, 121:123-131.

34. Veliz LP, Gonzalez FG, Duling BR, Saez JC, Boric MP: Functional role of gap junctions in cytokine-induced leukocyte adhesion to endothelium in vivo. Am J Physiol Heart Circ Physiol 2008, 295:H1056-H1066.

35. Parthasarathi $\mathrm{K}$, Ichimura $\mathrm{H}$, Monma $\mathrm{E}$, et al: Connexin 43 mediates spread of Ca2+-dependent proinflammatory responses in lung capillaries. J Clin Invest 2006, 116:2193-2200.

36. Chadjichristos $C E$, Morel $S$, Derouette JP, et al: Targeting connexin 43 prevents platelet-derived growth factor-BB-induced phenotypic change in porcine coronary artery smooth muscle cells: Circ Res. United States 2008, 653-660

37. Dussaule JC, Guerrot D, Huby AC, et al: The role of cell plasticity in progression and reversal of renal fibrosis. Int J Exp Pathol 2011.

38. Galichon P, Hertig A: Epithelial to mesenchymal transition as a biomarker in renal fibrosis: are we ready for the bedside? Fibrogenesis Tissue Repair 2011, 4:11.

39. Kriz W, Kaissling B, Le Hir M: Epithelial-mesenchymal transition (EMT) in kidney fibrosis: fact or fantasy? I Clin Invest 2011, 121:468-474

40. Piera-Velazquez S, Li Z, Jimenez SA: Role of Endothelial-Mesenchymal Transition (EndoMT) in the Pathogenesis of Fibrotic Disorders. Am J Pathol 2011, 179:1074-1080.

41. Zeisberg EM, Tarnavski O, Zeisberg M, et al: Endothelial-to-mesenchymal transition contributes to cardiac fibrosis. Nat Med 2007, 13:952-961.

42. Kizu A, Medici D, Kalluri R: Endothelial-mesenchymal transition as a novel mechanism for generating myofibroblasts during diabetic nephropathy. Am J Pathol 2009, 175:1371-1373.

43. Zeisberg EM, Potenta SE, Sugimoto H, Zeisberg M, Kalluri R: Fibroblasts in kidney fibrosis emerge via endothelial-to-mesenchymal transition. J Am Soc Nephrol 2008, 19:2282-2287.

44. Li J, Qu X, Yao J, et al: Blockade of endothelial-mesenchymal transition by a Smad3 inhibitor delays the early development of streptozotocininduced diabetic nephropathy. Diabetes 2010, 59:2612-2624.
45. Basile DP, Friedrich JL, Spahic J, et al: Impaired endothelial proliferation and mesenchymal transition contribute to vascular rarefaction following acute kidney injury. Am J Physiol Renal Physiol 2011, 300:F721-733.

46. Melo-Filho NM, Belmiro CL, Gonçalves RG, et al: Fucosylated chondroitin sulfate attenuates renal fibrosis in animals submitted to unilateral ureteral obstruction: a P-selectin-mediated event? Am J Physiol Renal Physiol 2010, 299:F1299-1307.

47. Choi DE, Jeong JY, Lim BJ, et al: Pretreatment of sildenafil attenuates ischemia-reperfusion renal injury in rats. Am J Physiol Renal Physiol 2009, 297:F362-370.

48. Asgeirsdóttir SA, Kamps JA, Bakker HI, et al: Site-specific inhibition of glomerulonephritis progression by targeted delivery of dexamethasone to glomerular endothelium. Mol Pharmacol 2007, 72:121-131.

49. Masuda Y, Shimizu A, Mori T, et al: Vascular endothelial growth factor enhances glomerular capillary repair and accelerates resolution of experimentally induced glomerulonephritis. Am J Pathol 2001, 159:599-608.

50. Chade AR, Zhu XY, Krier JD, et al: Endothelial progenitor cells homing and renal repair in experimental renovascular disease. Stem Cells 2010, 28:1039-1047.

51. Stokman G, Stroo I, Claessen N, Teske GJ, Florquin S, Leemans JC: SDF-1 provides morphological and functional protection against renal ischaemia/reperfusion injury. Nephrol Dial Transplant 2010, 25:3852-3859.

doi:10.1186/1755-1536-5-S1-S15

Cite this article as: Guerrot et al: Progression of renal fibrosis: the underestimated role of endothelial alterations. Fibrogenesis \& Tissue Repair 2012 5(Suppl 1):S15.

\section{Submit your next manuscript to BioMed Central and take full advantage of:}

- Convenient online submission

- Thorough peer review

- No space constraints or color figure charges

- Immediate publication on acceptance

- Inclusion in PubMed, CAS, Scopus and Google Scholar

- Research which is freely available for redistribution

Submit your manuscript at www.biomedcentral.com/submit
Biomed Central 